\title{
Erectile dysfunction post radical cystectomy. The role of early rehabilitation with pharmacotherapy in nerve sparing and non-nerve sparing group: A randomized, clinical trial
}

\author{
Mohamad Moussa $^{1}$, Athanasios G. Papatsoris ${ }^{2}$, Mohamed Abou Chakra ${ }^{3}$, Athanasios Dellis ${ }^{4}$ \\ ${ }^{1}$ Urology Department, Zahraa Hospital, University Medical Center, Beirut, Lebanon; \\ $22^{\text {nd }}$ Department of Urology, School of Medicine, Sismanoglio Hospital, National and Kapodistrian University of Athens, Athens, \\ Greece; \\ ${ }^{3}$ Faculty of Medical Sciences, Department of Urology, Lebanese University, Beirut, Lebanon; \\ ${ }^{4}$ Department of Urology/General Surgery, Areteion Hospital, Athens, Greece.
}

\begin{abstract}
Summary Objectives: No clinical studies testing erectile function $(E F)$ post radical cystectomy (RC) were done. Our objective was to assess the effect of early pharmacologic therapy after RC using intracorporeal injection (ICI), phosphodiesterase inhibitor (PDE5i) and PDE5i+ICI. Materials and Methods: In our randomized, double-blinded study, we prospectively enrolled 160 potent male patients with invasive bladder cancer. Patients were operated by RC using the nerve-sparing (NS) or non-nerve sparing (NNS) technique. They were treated since 1 month postoperatively by different regimens (PDE5i vs. ICI vs. ICI+PDE5i). Patients were evaluated using the international index of erectile function questionnaire and were followed up regularly at 1, 3, 6, and 12 months using the same parameters.

Results: One month after therapy, the mean of EF domain improved in both NS and NNS group. In the NNS group, in patients treated with ICI alone and ICI+PDE5i, the EF domain at 12 months moved to the moderate and to the mild category respectively. In patients treated by the NS approach, the mean value remained in the mild category with or without therapy. Conclusions: Early pharmacotherapy since one-month post RC using ICI and a combination of ICI+PDE5i can improve the erectile function of patients operated with a NNS approach.
\end{abstract}

KEY WORDS: Erectile dysfunction; Early rehabilitation; Radical cystectomy; Nerve-sparing; Non nerve-sparing.

Submitted 21 September 2020; Accepted 27 October 2020

\section{INTRODUCTION}

Radical cystectomy (RC) remains the standard of therapy for high-grade invasive bladder cancer and some categories of superficial bladder cancer (1). Sexual dysfunction after RC may be related to multiple factors including surgical trauma to the neurovascular bundle, psychological stress, and type of urinary diversion. RC with urinary diversion significantly affects sexual functioning. The recovery percentage of the erectile function was $49 \%$ at 3 years and $79 \%$ at 5 years $(2,3)$. We performed a prospective study testing the erectile function of 160 potent males operated by RC with nerve-sparing and none-nerve sparing technique, dividing them into groups that were treated early since 1 month after surgery by different drugs: intracorporeal injection (ICI), phosphodiesterase inhibitor (PDE5i) and a combination of both drugs. The objective of this study was to assess the erectile function (EF) in different subgroups of patients who were treated early postoperatively by comparing the preoperative "international index of erectile function (IIEF) questionnaire" results with postoperative results to test the effect of early rehabilitation strategy on erectile dysfunction. There is no evidence in the current literature regarding sexual function recoverability after RC focusing and, in particular, early rehabilitation strategy of erectile function and its effect on other domains of male sexual function (orgasmic function, sexual desire, intercourse satisfaction, and overall satisfaction) in the nonnerve and nerve sparing group of patients by passing the early phase of recovery.

\section{Material and Methods}

Study design E participants

This study was a randomized, double-blinded, clinical trial that evaluated the role of early pharmacologic therapy for the treatment of erectile dysfunction post RC. Between February 2014 and 2019, 160 potent male patients with non-metastatic invasive bladder cancer who were operated with radical cystectomy were included in the study. Patients were divided into 4 different groups: Group A including 40 patients who received no treatment. Group B including 40 patients who were treated by intracorporeal injection (ICI) of prostaglandin E1 (PGE1) at a dosage of 20 micrograms 2 times per week) after one month of surgery to 1 year. Group C including 40 patients who were treated by ICI+PDE5-i after one month of surgery to 1 year. Group D including 40 patients who were treated by PDE5i: Sildenafil, $50 \mathrm{mg}$ after one month of surgery to 1 year.

The patients were randomly allocated to each group based on the type of surgery performed. In each group, 20 patients were operated using the nerve sparing (NS) technique and 20 other patients operated using the non- 
nerve sparing (NNS) technique. All patients included in the study fulfilled the following criteria: potent male, sexually active, tumors muscle invasive T3 or less (T2a, T2b, T1High grade), N0, M0, age 45-69, not receiving neoadjuvant chemotherapy.

The patients and treating clinicians were blinded to the treatment allocation. Follow up visits were noted by another urologist within the same department.

\section{Sample size}

Based on previous studies, the difference in the mean change in the IIEF score between the two groups (NS and NNS) was 11.7. We conservatively set this value as 11. When a two-tailed test with a test power of $90 \%$ and a significance level of 5\% was used, 80 patients are required in each group. For 4 treatment subgroups, the total sample size required for this study was estimated to be 160 patients.

\section{Study intervention}

The same urologist performed the RC+ Ileal conduit (90 patients) or neobladder (70 patients) with or without nerve sparing technique. $\mathrm{RC}$ was done with preservation of the neurovascular bundles as described by Walsh (5). Three groups of patients were treated since one month post operatively with PDE5i, ICI, PDE5i+ICI for a period of 1 year where they were followed.

The study was performed at Zahraa Hospital, University Medical Center in Beirut which is an academic, tertiary care hospital with 300 beds. The Urology Department contains 22 beds; it is staffed by 8 urologists. The study was approved by our Institutional Review Board. Our study adheres to CONSORT guidelines.

Descriptive tables of means and standard deviations were computed for different combinations.

\section{Outcome measurements}

Erectile function was assessed by IIEF- score before and after surgery at 1, 3, 6, 12 months postoperatively.

Preoperative EF was tested using the IIEF questionnaire (4). The degree of the erection was categorized on the basis of the EF domain (six questions regarding $\mathrm{EF}$ ) of IIEF questionnaire and classified as mild ED (score 1725), moderate ED (score 11-16), or severe dysfunction (score $<10$ ); patients with score $>26$ were considered as not having erectile dysfunction.

\section{Ethics statement}

The study was approved by our hospital IRB (approval No. 2014.12). Informed consent was confirmed by the IRB. Written informed consent was obtained from each participant.

\section{Statistical analysis}

Statistical analyses for estimated marginal means were performed using a mixed ANOVA with repeated measures. This test determines the interactions between dependent and independent variables.

A mixed ANOVA with repeated measures was run on a sample of 160 patients to examine the effect of the treatment group of 4 levels (No therapy, PDE5-I, ICI, ICI+PDE5-I), the type of surgery of 2 levels (NS and
NNS) and the time points of assessment of 5 levels (preoperatively time, 1 month postoperatively, 3 months postoperatively, 6 months postoperatively, 12 months postoperatively) (3 independent variables) on the erectile function.

The normal distribution and the homogeneity of variances assumptions for each combination of the groups were checked using respectively the Shapiro-wilk tests of normality and the Levene's Test of Equality of Error Variances. The data was processed using the Statistics software SPSS (Statistical Package for Social Sciences) Version 19.0. A p value of 0.05 was considered statistically significant.

\section{Results}

Tablel summarizes the clinicopathologic characteristics of the patients from which reported data were obtained. Median patient age in the NS group was 62 vs. 61 in NNS group and both groups had the same comorbidities. Orthotopic neobladder was performed for 70 patients (45 patients in the NS group and 25 patients in the NNS group). Ileal conduit urinary diversion was performed in 90 patients (35 of them in the NS group and 55 others in the NNS group).

The mean preoperative EF domain in NNS group was 20.7 wherein in NS group was 21.4. After 1 month it was 6 in NNS group wherein in NS group was 9.8 (severe erectile dysfunction).

The mean values of $\mathrm{EF}$ domains differed in each subgroup of the 2 major groups (NS vs. NNS). After 1 month from surgery, NNS groups with no therapy, PDE5i, ICI and ICI+PDE5 I showed all the same value of 6. In NS groups with no therapy, PDE5i, ICI and ICI+PDE5 I subgroup mean value was $12.5,10.5,9$ and 7.5 respectively.

The mean value of EF domains in NNS group remained the same with no therapy and PDE5i at 3, 6, 12 months with a value of 6 , whereas in the ICI subgroup it increased to 13.5 at 4 months remaining constant at 6 and 12 months and in the ICI+PDE5i association, it increased the mild range of 18 at 12 months. The mean of EF domains in NS group increased to become in all subgroups in the mild range. Those results are illustrated in details in Table 2.

When assessing the difference of means between treatment subgroup, we found only a statistical significance ( $\mathrm{p}<0.05$ ) when comparing the PDE5i subgroup with other treatment subgroups (ICI, ICI+PDE5i), where no statistical significance was found when comparing ICI

\section{Table 1.}

Clinicopathologic characteristics and comorbidities of the NS and NNS group.

\begin{tabular}{|lll|}
\hline & NS & NNS \\
\hline pT 1 HG & 25 & 31 \\
\hline $\mathrm{T} 2 \mathrm{a}$ & 21 & 20 \\
\hline $\mathrm{T} 2 \mathrm{~b}$ & 19 & 9 \\
\hline $\mathrm{T} \mathrm{a} a$ & 15 & 20 \\
\hline Hypertension & 20 & 33 \\
\hline Diabetes & 13 & 10 \\
\hline NS: nerve-sparing; NNS: non nerve sparing; HG: high grade. & & \\
\hline
\end{tabular}


Table 2.

Mean of erectile function domains in the NS and NNS group divided into treatment subgroups.

\begin{tabular}{|l|lc|cccc|}
\hline & & \multicolumn{4}{|c|}{ Treatment subgroup } \\
& Type of surgery & None & PDE5-I & ICI & ICI+PDE5-I \\
\hline EF_Preop & NNS & $20.7 \pm 2.9$ & $22 \pm 2.2$ & $19.5 \pm 3$ & $20.5 \pm 2.9$ & $21 \pm 3.5$ \\
& NS & $21.45 \pm 2.8$ & $24 \pm 1.4$ & $21.3 \pm 3.2$ & $21 \pm 3.6$ & $19.5 \pm 3$ \\
\hline 1 month postop & NNS & $6 \pm 0$ & $6 \pm 0$ & $6 \pm 0$ & $6 \pm 0$ & $6 \pm 0$ \\
& NS & $9.8 \pm 3.2$ & $12.5 \pm 3.3$ & $10.5 \pm 3.1$ & $9 \pm 3.5$ & $7.5 \pm 3$ \\
\hline 3 months postop & NNS & $10.3 \pm 1.4$ & $6 \pm 0$ & $6 \pm 0$ & $13.5 \pm 3$ & $15.8 \pm 2.6$ \\
& NS & $10.3 \pm 3.3$ & $14.3 \pm 2.2$ & $14.5 \pm 5.7$ & $18.8 \pm 4.9$ & $17.5 \pm 0.6$ \\
\hline 6 months postop & NNS & $10.7 \pm 0.9$ & $6 \pm 0$ & $6 \pm 0$ & $13.5 \pm 3$ & $17.3 \pm 0.9$ \\
& NS & $19.1 \pm 3.6$ & $17 \pm 4.9$ & $18.3 \pm 4.5$ & $21.8 \pm 2.6$ & $19.3 \pm 2.5$ \\
\hline 12 months postop & NNS & $10.8 \pm 1.1$ & $6 \pm 0$ & $6 \pm 0$ & $13.5 \pm 3$ & $18 \pm 1.6$ \\
& NS & $20.9 \pm 2.9$ & $19.5 \pm 3.1$ & $19.8 \pm 2.9$ & $22 \pm 2.7$ & $22.3 \pm 2.9$ \\
\hline PDE5-I: Phosphodiesterase inhibitor; ICl: Intracavernosal injection; EF: erectile function; NS: nerve-sparing; \\
NNS: non nerve-sparing; post op: post operative period. \\
\hline \multicolumn{7}{|c|}{}
\end{tabular}

Table 3.

Mean of erectile function domains compared between treatment subgroups.

\begin{tabular}{|l|c|c|c|c|c|}
\hline $\begin{array}{l}\text { Treatment } \\
\text { subgroup 1 (G1) }\end{array}$ & $\begin{array}{c}\text { Treatment } \\
\text { subgroup 2 (G2) }\end{array}$ & $\begin{array}{c}\text { Mean } \\
\text { G1 }\end{array}$ & $\begin{array}{c}\text { Mean } \\
\text { G2 }\end{array}$ & $\begin{array}{c}\text { Mean } \\
\text { difference (G1-G2) }\end{array}$ & P-value \\
\hline None & PDE5-I & 13.325 & 12.775 & 0.55 & 1 \\
& ICI & & 15.95 & -2.625 & 0.09 \\
& ICI+PDE5-I & & 16.4 & -3.075 & $0.03^{*}$ \\
\hline PDE5-I & ICI & 12.775 & 15.95 & -3.175 & $0.02^{*}$ \\
& ICI+PDE5-I & & 16.4 & -3.625 & $0.008^{*}$ \\
\hline ICI & ICI+PDE5-I & 15.95 & 16.4 & -0.45 & 1 \\
\hline PDE5-I: Phosphodiesterase inhibitor; ICl: Intracavernosal injection; None: patients not giving any treatment. \\
\hline
\end{tabular}

vs. ICI+PDE5i. Those results are illustrated in Table 3. Overall results demonstrated that means differ significantly according to the type of surgery, with 17.5 in NS to 1.72 in NNS group $(\mathrm{p}<0.001)$. Results of the treatment subgroups according to type of surgery are illustrated in Figure 1.

We also assessed the differences of each treatment subgroup according to the type of surgery. We found statistical differences in the NNS group, when we compared no therapy with ICI or ICI+PDE5i whereas no difference was noted when comparing no therapy with PDE5i or comparing ICI with ICI+PDE5i. We didn't find any statistical significance in the NS group when comparing all

Figure 1.

Two-way interactions for erectile function domain: Treatment subgroups $\times$ type of surgery.

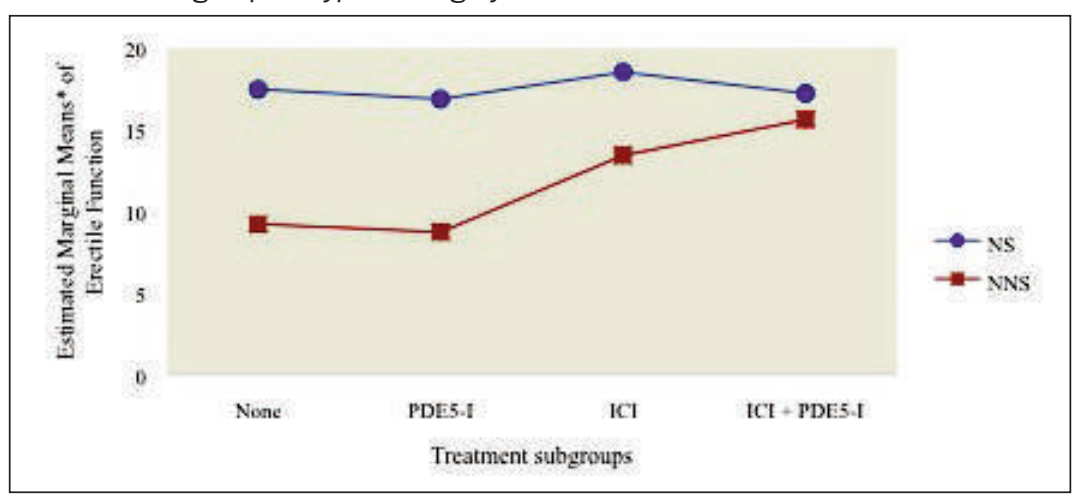

Table 4.

Direct erectile function domain comparison between patient's subgroups according to the type of surgery.

\begin{tabular}{|c|c|c|c|c|c|c|}
\hline $\begin{array}{l}\text { Type of } \\
\text { surgery }\end{array}$ & $\begin{array}{l}\text { Treatment } \\
\text { subgroup } 1 \text { (G1) }\end{array}$ & $\begin{array}{c}\text { Treatment } \\
\text { subgroup } 2 \text { (G2) }\end{array}$ & $\begin{array}{c}\text { Mean } \\
\text { G1 }\end{array}$ & $\begin{array}{c}\text { Mean } \\
\text { G2 }\end{array}$ & $\begin{array}{c}\text { Mean } \\
\text { difference (G1-G2) }\end{array}$ & P-value \\
\hline NNS & $\begin{array}{l}\text { None } \\
\text { PDE5-I } \\
\text { ICI }\end{array}$ & $\begin{array}{c}\text { PDE5-I } \\
\text { ICI } \\
\text { ICI+PDE5-I } \\
\text { ICI } \\
\text { ICI+PDE5-I } \\
\text { ICI+PDE5-I }\end{array}$ & $\begin{array}{l}8.7 \\
13.4\end{array}$ & $\begin{array}{c}8.7 \\
13.4 \\
15.6 \\
13.4 \\
15.6 \\
15.6\end{array}$ & $\begin{array}{l}0.5 \\
-4.2 \\
-6.4 \\
-4.7 \\
-6.9 \\
-2.2\end{array}$ & $\begin{array}{c}1 \\
0.039^{*} \\
0.001^{*} \\
0.016^{*} \\
<0.0001^{*} \\
0.8\end{array}$ \\
\hline$\overline{N S}$ & $\begin{array}{l}\text { None } \\
\text { PDE5-I } \\
\text { IC| }\end{array}$ & $\begin{array}{c}\text { PDE5-I } \\
\text { ICI } \\
\text { ICI+PDE5-I } \\
\text { ICI } \\
\text { ICI+PDE5-I } \\
\text { ICI+PDE5-I }\end{array}$ & $\begin{array}{l}16.85 \\
18.5\end{array}$ & $\begin{array}{c}16.85 \\
18.5 \\
17.2 \\
18.5 \\
17.2 \\
17.2\end{array}$ & $\begin{array}{c}0.6 \\
-1.05 \\
0.25 \\
-1.65 \\
-0.35 \\
1.3\end{array}$ & $\begin{array}{l}1 \\
1 \\
1 \\
1 \\
1 \\
1\end{array}$ \\
\hline
\end{tabular}

the subgroups between them; those results are illustrated in Table 4.

\section{Discussion}

ED occurs frequently in patients operated by RC. Walsh et al. demonstrated that injury to the neurovascular bundles may contribute to impotence following radical prostatectomy (RP) (5). Based on those findings in 1984, Walsh used a surgical technique that preserves the branches of the pelvic plexus for the preservation of potency and this technique was called a nerve-sparing technique (6).

Different sexual-preserving techniques have been described with a different effect on functional and oncological outcomes. The potency rates of capsule sparing cystectomy vs. nerve-sparing cystectomy were not significantly different (50\% vs. 40\%) as reported by Jacobs et al. (7). A study conducted by Colombo et al. evaluating 3 techniques of nerve sparing cystectomy showed a significant difference in sexual function preservation using the capsule sparing and seminal vesicle sparing vs. nerve sparing ( $91.6 \%$ vs. 84.25 vs $28.5 \%$ ) but this study was limited due to a low number of patients operated (8). However, those techniques might be not oncologically safe with high metastasis rate (9). In addition, around half of the cystectomy specimens may contain urothelial carcinoma; cancer control may be compromised by leaving a part of the prostate in the capsule sparing technique (10). Therefore, both nerve-sparing cystectomy and prostate capsule sparing cystectomy appear to offer better urinary and sexual function in properly selected patients. More randomized clinical trials are necessary to use those techniques safely in bladder cancer era.

Several studies reported that the recovery of erectile function after nerve-sparing radical cystoprostatectomy is around $30 \%$ to $80 \%$ of cases $(2,3,11,12)$. 
There is a clear correlation between the age of the patient when operated and the erectile function recovery following nerve-sparing cystectomy as demonstrated by Schoenberg et al., who showed that the recovery rate of sexual function was the lowest in men 70-79 year old after 10 years of experience with nerve-sparing radical cystectomy (13). Younger age was associate with better sexual function after radical cystectomy then older patients (14-16)

Impotence and lack of sexual desire were related to radical radiotherapy for bladder cancer with only 34\% male patients sexually active after radiation therapy (17).

The type of urinary diversion influence also the sexual activity after surgery where patients with ileal conduit diversion reported more erectile dysfunction $(18,19)$.

Due to the lack of studies addressing the best treatment for erectile dysfunction post radical cystectomy, the response to oral therapy was extrapolated from series on post prostatectomy erectile dysfunction management, where sildenafil is considered an effective treatment after radical retropubic prostatectomy when both neurovascular bundles have been preserved with response rate after nerve-sparing approach reaching $80 \%(20,21)$.

Cavernous smooth muscle apoptosis is one mechanism of impotence as tested in multiple experimental studies. Apoptosis of smooth muscle in the rat penis was more significant after bilateral neurectomy vs. unilateral neurectomy $(22,23)$. Prostaglandin E1 and PDE5i, if given in the early postoperative period could improve erections by providing intracavernosal oxygenation and limiting fibrosis within the corpora cavernosa (24).

PDE5i, if used as part of the early penile rehabilitation therapy after RP, could preserve intracorporeal smooth muscle content (25). Also, it had been shown that early use of PDE5i decreased the numbers of apoptotic cells and prevented apoptotic cell death in the penis following denervation (26). Zippe et al. demonstrated that sildenafil citrate cannot improve significantly erectile dysfunction after radical cystectomy: out of 22 patients taking sildenafil citrate post-surgery, only 2 (9\%) responded positively (18).

A study assessing the optimal time for intracavernous prostaglandin El administration after non-sparing RP found that early use of prostaglandin injection since 1 month postoperatively could promote the best response but increased the complication rate (27). Montorsi et al. conducted a study evaluating the early intracavernous injections of alprostadil after nerve-sparing RP. They reported that early injections after 6 months of follow up induced a significant improvement of the spontaneous erections where $67 \%$ of the group treated by early intracavernous aplrostadil injection recovered sufficient spontaneous erections for sexual intercourse (28).

Padma-Nathan et al. demonstrated that early administration of sildenafil since one month after bilateral nervesparing RP may help in spontaneous erectile function recovery (29). Cavernous neurotomy may cause cavernous fibrosis and dysfunction by increasing expression of TGF-bl, and collagen I and III protein. This may explain why patients operated by nerve-sparing approach may respond partially to pharmacologic therapies for erectile dysfunction. Some new techniques using intraoperative cavernous nerve stimulation may help in nerve preservation surgeries although those techniques need more investigations (30).

There is widespread usage of Vacuum therapy (VT) as part of the penile rehabilitation after RP. The underlying hypothesis is that the artificial induction of erections shortly after surgery facilitates tissue oxygenation, reducing cavernosal fibrosis in the absence of nocturnal erections (31). Yuan et al. assessed the effects of VT on erectile dysfunction (ED) in a rat model of bilateral cavernous nerve crush (BCNC) demonstrating that EF was improved with VT. VT reduced hypoxia-inducible factor- $1 \alpha$ (HIF$1 \alpha$ ) expression and apoptotic indices (AI) significantly compared with control. Animals exposed to VT had decreased transforming growth factor beta 1 (TGF- $\beta 1$ ) expression, increased smooth muscle/collagen ratios (32). A web-based survey of members of the International Society for Sexual Medicine (ISSM) and its regional affiliates was performed to assess their practice pattern of post-RP pharmacological penile rehabilitation. As part of the primary rehabilitation strategy post RP, $30 \%$ of responders used vacuum device, 95\% PDE5i and 75\% used ICI (33). In a pilot study, it was demonstrated that initiating the use of a VT protocol at 1 month after RP improves early sexual function and helps to preserve penile length (34). Studies have shown that vacuum erectile device therapy improves erectile function in 84$95 \%$ of patients and it is suggested that penile rehabilitation with VT should begin early after RP (35).

The post-RP ED depends on several factors, including patient age, preoperative potency status (baseline EF) and comorbidities (36). Preoperative potency is of utmost importance, since patients who complain of some degree of ED or patients who already use PDE5-is prior to surgery are at higher risk of developing severe ED postoperatively, regardless of the surgical technique used (37).

The main goals of prostate capsule sparing cystectomy are to improve erectile function. Eleven published series of prostate sparing cystectomy report $80 \%$ or greater rates of potency preservation, and 6 describe $90 \%$ or greater preservation (38). Jacobs et al. performed a singleinstitution trial of bladder cancer patients. No significant difference in functional results were found in men randomized to prostate capsule sparing or nerve-sparing cystectomy with neobladder creation. Mertens et al. reported a 20-year single-center experience of prostate sparing cystectomy for bladder cancer showing that erectile function was intact in $89.7 \%$ of patients (39).

Back to our results, the mean of EF domain after 1 month of surgery was in the category of severe erectile dysfunction in both group NS and NNS, when the treatment was started after 1 month. The mean value improved in both groups to become at 12 months in the moderate and mild categories in patient treated with ICI and ICI+PDE5i in the NNS group. In other hands, in patients treated by the NS approach, the mean was in the mild category when they were treated by ICI or $\mathrm{ICI}+\mathrm{PDE} 5 \mathrm{i}$ or in the group not taking any drug. It is important to note that there was no difference between group not given any drug and PDE5i therapy alone in both groups (NS vs. NNS). It means that early therapy 
with PDE5i alone may be not effective in the early phase of erectile function recovery.

It is also necessary to mention that in the NNS group the mean improve to mild category if ICI and PDE5i were associated together and used early post-surgery.

It is critical to note the NS group results, showing that the mean of EF domain improved after 12 months to become in the same category in all subgroups, We cannot conclude if this improvement is due to early postoperative aid or related to neuropraxia phenomenon described previously.

In the absence of studies assessing erectile dysfunction post cystectomy in both groups (NS vs. NNS), our results were discussed in front of those obtained in radical prostatectomies patients.

The current study was limited by the methodology used to assess the erectile dysfunction. In fact, patients were evaluated using the international index of erectile function (IIEF) score only along a short period of follow up.

\section{Conclusions}

Erectile dysfunction is common after radical cystectomy surgery; it has a great impact on the quality of life of patients. Early rehabilitation plans using pharmacotherapy since one month after surgery with ICI and a combination of ICI+PDE5i can improve erectile function in patients operated with non-nerve sparing approach but not with nerve sparing approach. An early rehabilitative strategy with pharmacotherapy may be offered to patients undergoing a non nerve-sparing procedure in order to enhance the recovery of erectile function and improve all other domains. More studies addressing the early rehabilitative strategy of none nerve-sparing radical cystectomies are needed to confirm our findings.

\section{References}

1. Stein JP, Skinner DG. Results with radical cystectomy for treating bladder cancer: a 'reference standard' for high-grade, invasive bladder cancer. BJU Int. 2003; 92:12-7.

2. Schlegel PN, Walsh PC. Neuroanatomical approach to radical cystoprostatectomy with preservation of sexual function. J Urol. 1987; 138:1402-6.

3. Miyao N, Adachi $H$, Sato $Y$, et al. Recovery of sexual function after nerve-sparing radical prostatectomy or cystectomy. Int $J$ Urol.2001; 8:158-64.

4. Rosen RC, Riley A, Wagner G, et al. The International Index of Erectile Function (IIEF): a multidimensional scale for assessment of erectile dysfunction. Urology. 1997; 49:822-830.

5. Walsh PC, Donker PJ. Impotence following radical prostatectomy: insight into etiology and prevention. J Urol 1982; 128:492-497.

6. Walsh PC, Mostwin JL. Radical prostatectomy and cystoprostatectomy with preservation of potency. Results using a new nerve-sparing technique. Br J Urol 1984; 56:694-697.

7. Jacobs BL, Daignault S, Lee CT, et al. Prostate capsule sparing versus nerve sparing radical cystectomy for bladder cancer: results of a randomized, controlled trial. J Urol. 2015; 193:64-70.

8. Colombo R, Pellucchi F, Moschini M, et al. Fifteen-year singlecentre experience with three different surgical procedures of nerve- sparing cystectomy in selected organconfined bladder cancer patients. World J Uro.l 2015; 33:1389-95.

9. Botto H, Sebe P, Molinie V, et al. Prostatic capsule- and seminalsparing cystectomy for bladder carcinoma: initial results for selected patients. BJU Int. 2004; 94:1021-5.

10. Revelo MP, Cookson MS, Chang SS, et al. Incidence and location of prostate and urothelial carcinoma in prostates from cystoprostatectomies: implications for possible apical sparing surgery. $J$ Urol. 2004; 171:646.

11. Martis G, D'Elia G, Diana M, et al. Prostatic capsule- and nerve-sparing cystectomy in organ-confined bladder cancer: preliminary results. World J Surg. 2005; 29:1277-1281.

12. Horenblas S, Meinhardt W, Ijzerman W, Moonen LF. Sexuality preserving cystectomy and neobladder: initial results. J Urol. 2001; 166: 837-840.

13. Schoenberg MP, Walsh PC, Breazeale DR, et al. Local recurrence and survival following nerve sparing radical cystoprostatectomy for bladder cancer: 10-year followup. J Urol. 1996; 155:490-494.

14. Bjerre BD, Johansen C, Steven K. Sexological problems after cystectomy: bladder substitution compared with ileal conduit diversion. A questionnaire study of male patients. Scand J Urol Nephrol. 1998; 32:187-193.

15. Botto H, Sebe P, Molinie V, et al. Prostatic capsule- and seminal-sparing cystectomy for bladder carcinoma: initial results for selected patients. BJU Int. 2004; 94:1021-1025.

16. Kessler TM, Burkhard FC, Perimenis P, et al. Attempted nerve sparing surgery and age have a significant effect on urinary continence and erectile function after radical cystoprostatectomy and ileal orthotopic bladder substitution. J Urol. 2004; 172:1323-1327.

17. Fokdal L, Høyer M, Meldgaard P, von der Maase H. Long-term bladder, colorectal, and sexual functions after radical radiotherapy for urinary bladder cancer. Radiother Oncol. 2004; 72:139-145.

18. Zippe CD, Raina R, Massanyi EZ, et al. Sexual function after male radical cystectomy in a sexually active population. Urology. 2004; 64:682-685

19. Hobisch A, Tosun K, Kinzl J, et al. Quality of life after cystectomy and orthotopic neobladder versus ileal conduit urinary diversion. World J Urol. 2000; 18:338-44.

20. Zagaja GP, Mhoon DA, Aikens JE, Brendler CB. Sildenafil in the treatment of erectile dysfunction after radical prostatectomy. Urology. 2000; 56: 631-634.

21. Zippe CD, Jhaveri FM, Klein EA, et al. Role of Viagra after radical prostatectomy. Urology. 2000; 55:241-245.

22. User HM, Hairston JH, Zelner D, et al. Penile weight and cell subtype specific changes in a postradical prostatectomy model of erectile dysfunction. J Urol. 2003; 169:1175-1179.

23. Klein LT, Miller MI, Buttyan R, et al. Apoptosis in the rat penis after penile denervation J Urol. 1997; 158:626-630.

24. $R$. Wang. Penile rehabilitation after radical prostatectomy: where do we stand and where are we going? J Sex Med. 2007; 4:1085-97.

25. Schwartz EJ, Wong P, Graydon RJ. Sildenafil preserves intracorporeal smooth muscle after radical retropubic prostatectomy. J Urol. 2004; 171:771-4.

26. La Vignera S, Condorelli R, D'Agata R, et al. Dysfunction of the endothelial-platelet pathway in patients with erectile dysfunction before and after daily treatment with tadalafil. Andrologia. 2012; 44:152-156. 
27. Gontero P, Fontana F, Bagnasacco A, et al. Is there an optimal timing for intracavernous PGE1 rehabilitation following nonnerve sparing radical prostatectomy Results from an hemodynamic perspective study J Urol. 2003; 169:2166-2169.

28. Montorsi F, Guazzoni G, Strambi LF, et al. Recovery of spontaneous erectile function after nerve-sparing radical retropubic prostatectomy with and without early intracavernous injections of alprostadil: results of a prospective, randomised trial. J Urol. 1997; 158:1408-1410.

29. Padma-Nathan E, Mc Cullough, AR, Giuliano F. Postoperative nightly administration of sildenafil citrate significantly improves the return of normal spontaneous erectile function after bilateral nervesparing radical prostatectomy. J Urol. 2003; 4(Suppl):375.

30. Klotz L. Cavernosal nerve mapping: current data and applications. British Journal of Urology International. 2004; 93:9-13.

31. Yuan J, Hoang AN, Romero CA, et al. Vacuum therapy in erectile dysfunction--science and clinical evidence Int J Impot Res. 2010; 22:211-9.

32. Yuan J, Lin H, Li P, et al. Molecular mechanisms of vacuum therapy in penile rehabilitation: a novel animal study. Eur Urol. 2010; 58:773-80.
33. Teloken P, Mesquita G, Montorsi F, Mulhall J. Post-radical prostatectomy pharmacological penile rehabilitation: practice patterns among the international society for sexual medicine practitioners. J Sex Med. 2009; 6:2032-8.

34. Köhler TS, Pedro R, Hendlin K, et al. A pilot study on the early use of the vacuum erection device after radical retropubic prostatectomy. BJU Int. 2007; 100:858-62.

35. Lin $H$, Wang $R$. The science of vacuum erectile device in penile rehabilitation after radical prostatectomy. Transl Androl Urol. 2013; 2:61-66.

36. Saleh A, Abboudi H, Ghazal-Aswad M, et al. Management of erectile dysfunction post-radical prostatectomy. Res Rep Urol. 2015; 7:19-33.

37. Gallina A, Salonia A, Briganti A, et al. Prevention and management of postprostatectomy erectile dysfunction. Eur Urol Supplements. 2009; 8:80-7.

38. Klotz L. Prostate capsule sparing radical cystectomy: oncologic safety and clinical outcome. Ther Adv Urol. 2009; 1:43-50.

39. Mertens LS, Meijer RP, de Vries RR, et al. Prostate sparing cystectomy for bladder cancer: 20-year single center experience. J Urol. 2014; 191:1250-5.

\section{Correspondence}

Mohamad Moussa, MD

mohamadamoussa@hotmail.com

Urology Department, Zahraa Hospital, University Medical Center, Beirut (Lebanon)

Athanasios G. Papatsoris, MD

agpapatsoris@yahoo.gr

$2^{\text {nd }}$ Department of Urology, School of Medicine, Sismanoglio Hospital,

National and Kapodistrian University of Athens, Athens (Greece)

Mohamed Abou Chakra, MD (Corresponding Author)

mohamedabouchakra@hotmail.com

Faculty of Medical Sciences, Department of Urology, Lebanese University,

Beirut, (Lebanon), 1108

Athanasios Dellis, MD

aedellis@gmail.com

Department of Urology/General Surgery, Areteion Hospital, Athens (Greece) 